\title{
Transcriptome profiling and pathway analysis of the effects of mono-(2-ethylhexyl) phthalate in mouse Sertoli cells
}

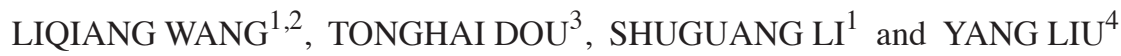 \\ ${ }^{1}$ Key Laboratory of Public Health Safety of The Ministry of Education, School of Public Health, Fudan University, \\ Shanghai 200032; ${ }^{2}$ College of Medical Technology, Shanghai University of Medicine \& Health Sciences, \\ Shanghai 201318; ${ }^{3}$ Department of Microbiology and Microbial Engineering, School of Life Sciences, \\ Fudan University, Shanghai 200438; ${ }^{4}$ Shanghai Institute of Quality Inspection and Technical Research, \\ National Quality Supervision and Inspection Center for Food Products (Shanghai), Shanghai 200233, P.R. China
}

Received May 16, 2018; Accepted January 28, 2019

DOI: $10.3892 /$ etm.2019.7239

\begin{abstract}
Phthalates are confirmed to have toxic effects on the reproductive system and are likely to have further damaging actions in humans. The present study explored the molecular mechanisms of the toxic effect of mono-(2-ethylhexyl) phthalate (MEHP) on mouse Sertoli cells. Cell apoptosis and proliferation assays were used to assess the effects of MEHP on the TM4 Sertoli cell line derived from mouse testes. TM4 cells were treated with two doses of MEHP or left untreated as a control group, followed by RNA extraction and analysis using high-throughput transcriptome sequencing technology. The gene expression profile obtained was then subjected to a bioinformatics analysis to explore the molecular mechanisms of reproductive toxicity. The results revealed that 528 and 269 genes were upregulated in the high- and low-dose MEHP groups of cells compared with the control group, while 148 and 173 genes were downregulated. Gene ontology (GO) analysis indicated that the differently expressed genes were associated with the GO term 'extracellular region' of the cellular component domain in the high and low MEHP groups. Compared with the control group, eight common pathway changes were identified in the high- and low-dose MEHP groups, including 'terpenoid backbone biosynthesis'. Reverse transcription-quantitative polymerase chain reaction analysis
\end{abstract}

Correspondence to: Professor Shuguang Li, Key Laboratory of Public Health Safety of The Ministry of Education, School of Public Health, Fudan University, 130 Dongan Road, Shanghai 200032, P.R. China

E-mail: leeshuguang@fudan.edu.cn

Dr Yang Liu, Shanghai Institute of Quality Inspection and Technical Research, National Quality Supervision and Inspection Center for Food Products (Shanghai), 381 Cangwu Road, Shanghai 200233, P.R. China

E-mail: liuyanghai28@126.com

Key words: mono-(2-ethylhexyl) phthalate, Sertoli cells, transcriptome, reproductive toxicity was used to validation, and hermetic effects were observed for certain genes. These results provide an important basis and experimental data for further research into the mechanisms of phthalate-induced toxicity.

\section{Introduction}

Phthalates are a group of industrial chemicals that are used as plasticizers, lubricants and solvents, and impart favorable characteristics to various consumer products, including food packaging, pharmaceuticals, medical devices, construction materials and household furnishings (1). In 2006, the total production of plasticizer phthalates was $\sim 4.06$ million metric tons (2). As a result of ubiquitous exposure, phthalates and their metabolites are detected in biological samples from humans, including urine and serum (3). Individuals are continuously exposed to phthalates via ingestion, inhalation, intravenous injection and skin absorption, beginning in utero, and dietary intake from contaminated foods is thought to be the most important means of exposure (4-6). Di-(2-ethylhexyl) phthalate (DEHP) is the most abundant phthalate in the environment (7). Phthalates may provoke various adverse effects, including endocrine disruption, as well as developmental and reproductive dysfunction (8). The mechanisms of their toxic effects on the reproductive system have remained to be fully elucidated; however, certain effects are potentially associated with anti-androgenic activity (9).

The molecular mechanisms of phthalate toxicity have been heavily investigated; however, previous studies have only focused on one or several cellular and molecular biological processes, or the expression of a small number of genes/proteins. It is difficult to collate this information to systematically explain the molecular mechanisms of phthalate toxicity. In recent years, next-generation sequencing (NGS) technology has emerged to provide an effective method for high-throughput sequence determination, and has markedly improved the speed and efficiency of the identification of novel key genes or biomarkers $(10,11)$.

Previous studies have indicated that phthalates decrease the number of Sertoli cells per tubule in testis cross-sections in a dose dependent manner, promote disruption of cellular tight 
junction proteins (12), and decrease androgen receptor (13) and follicle-stimulating hormone (FSH) receptor expression. Sertoli cells appear to be the primary target of DEHP and mono-(2-ethylhexyl) phthalate (MEHP), an active metabolite of DEHP, in rodents (14-16). TM4 is an immature Sertoli cell line derived from the testis of an immature BALB/c mouse, and it is a particularly good model for investigating endocrine disruption, including the effects of phthalates, as the cells maintain the ability to respond to FSH stimulation and express androgen and estrogen receptors (17). Considering that NGS technology is recognized as a reliable method to determine mechanisms of drug-induced activity and to identify biomarkers (18), the present study performed whole-transcriptome sequencing of control- and MEHP-treated TM4 cells to identify differentially expressed genes (DEGs) and to provide novel insight into the toxic mechanisms of phthalates.

\section{Materials and methods}

Materials and chemicals. The TM4 normal mouse Sertoli cell line was obtained from the American Type Culture Collection (Manassas, VA, USA). Fetal bovine serum (FBS) was purchased from Thermo Fisher Scientific, Inc. (Waltham, MA, USA). MEHP standard reagent was obtained from Shanghai Aladdin Biochemical Technology Co., Ltd. (Shanghai, China). The Annexin V-fluorescein isothiocyanate (FITC)/propidium iodide (PI) apoptosis kit (cat. no. AD10) and Cell Counting Kit-8 (CCK-8; cat. no. CK04) were obtained from Dojindo Molecular Technologies, Inc. (Kumamoto, Japan). The TruSeq ${ }^{\circledR}$ RNA LT Sample Prep Kit v2 (cat. no. RS-122), ruSeq PE Cluster Kit v3-cBot-HS (cat. no. GD-401-3001) and TruSeq SBS Kit v3-HS (cat. no. FC-401) used for RNA library construction were obtained from Illumina, Inc. (San Diego, CA, USA). The SYBR Premix Ex Taq reagent kit (cat. no. RR420) and PrimeScript RT reagent kit (cat. no. RR037) were purchased from Takara Biotechnology Co., Ltd. (Dalian, China).

Cell culture. TM4 cells were cultured at $37^{\circ} \mathrm{C}$ with $5 \% \mathrm{CO}_{2}$ in Dulbecco's modified Eagle's medium supplemented with $10 \%$ FBS and $0.5 \%$ penicillin-streptomycin.

Apoptosis analysis. The cells $\left(>1 \times 10^{6}\right)$ were seeded on a 6-well cell culture plate. Following cell attachment at $60-70 \%$, MEHP dissolved in dimethylsufloxide (DMSO) was added. The final concentrations used were $1 \times 10^{-4}, 1 \times 10^{-5}$ and $1 \times 10^{-6} \mathrm{~mol} / 1$ ( $1 \% \mathrm{v} / \mathrm{v}$ DMSO). An equal volume of DMSO was added to the control group. Each treatment group was set up in three wells. After $48 \mathrm{~h}$, apoptosis was detected using a flow cytometer (FACS Calibur; Becton, Dickinson and Company, Franklin, Lakes, NJ, USA) according to the instructions of the Annexin V FITC/PI apoptosis kit.

Cell proliferation assay. TM4 cells were seeded in each well of a 96-well plate (3,000 cells per well) and incubated for $24 \mathrm{~h}$ at $37^{\circ} \mathrm{C}$ to allow for cell attachment. After $24 \mathrm{~h}$, cells were exposed to serially diluted concentrations of MEHP $\left(1 \times 10^{-3}\right.$, $5 \times 10^{-4}, 2.5 \times 10^{-4}, 1.25 \times 10^{-4}, 6.25 \times 10^{-5}, 3.13 \times 10^{-5}, 1.56 \times 10^{-5}$, $7.81 \times 10^{-6}, 3.91 \times 10^{-6}$ and $1.95 \times 10^{-6} \mathrm{mmol} / 1$; con 1 -con 10 , respectively). Each treatment group was set up in three wells. The cells were cultured in medium containing $10 \%$ FBS and $1 \%$
Table I. Results of the flow cytometric early apoptosis (\%) assay.

\begin{tabular}{lcccc}
\hline MEHP, mol $/ 1$ & $\mathrm{M}, \%\left(\mathrm{X}_{\min }, \mathrm{X}_{\max }\right)$ & $\mathrm{Q}(\%)$ & $\chi^{2}$ & $\mathrm{P}$-value \\
\hline 0 (control) & $0.39(0.34,0.45)$ & 0.06 & 7.42 & 0.06 \\
$1 \times 10^{-4}$ & $0.49(0.47,0.98)$ & 0.14 & & \\
$1 \times 10^{-5}$ & $0.48(0.20,0.55)$ & 0.16 & & \\
$1 \times 10^{-6}$ & $0.46(0.38,0.56)$ & 0.14 & & \\
\hline
\end{tabular}

Values are expressed as the $\mathrm{M}\left(\mathrm{X}_{\min }, \mathrm{X}_{\max }\right)$. M, median of early apoptotic cells; $\mathrm{X}_{\min }$, minimum value; $\mathrm{X}_{\max }$, maximum value; $\mathrm{Q}$, interquartile range; MEHP, mono-(2-ethylhexyl) phthalate.

$\mathrm{v} / \mathrm{v}$ DMSO. After 24 or $48 \mathrm{~h}$, the relative cell number was estimated using a CCK-8 proliferation kit. The absorbance at $450 \mathrm{~nm}$ was read using an Envision plate reader (PerkinElmer, Inc., Waltham, MA, USA) for cell counting.

RNA library construction and sequencing. Based on the results of the proliferation assay, TM4 cells were exposed to $1 \times 10^{-3}$ (con 1 ) and $2.5 \times 10^{-4}$ (con3) mmol/l MEHP for $48 \mathrm{~h}$. The cells were then subjected to transcriptome sequencing. Total RNA from TM4 cells in the treated and control group was isolated using TRIzol reagent (Thermo Fisher Scientific, Inc.). The RNA quantity and quality were examined using a Nanodrop 2000 instrument (Nanodrop Technologies; Thermo Fisher Scientific, Inc.) and electrophoresis, respectively. A total of three RNA libraries were constructed using Illumina TruSeq Stranded mRNA library prep kits according to the manufacturer's protocols and all sequencing was performed using the Illumina Hiseq2500 (Illumina, Inc.). The experimental results were processed using FastQC0.11.2 software (Babraham Bioinformatics, Cambridge, UK).

Reverse transcription-quantitative polymerase chain reaction $(R T-q P C R)$. DEGs were selected from the results of the transcriptome sequencing $(\mathrm{P}<0.005)$ and verified by RT-qPCR. Primers (details in Table II) were designed to span intron boundaries to avoid amplification of genomic DNA and to amplify all known isoforms of each gene based on the National Center for Biotechnology Information (NCBI) Reference Sequence Database (RefSeq; https://www.ncbi.nlm.nih.gov/refseq/). Total RNA from TM4 cells in the treated and control group was isolated using TRIzol reagent (Thermo Fisher Scientific, Inc.; cat. no. 15596026). RNA was transcribed into DNA using Transcriptor First Stand cDNA Synthesis kit (Roche Diagnostics, Basel, Switzerland; cat. no. 04897030001). The RT-Primers were Anchored-oligo(dT)18 Primer and Random Hexamer Primer. Primers were synthesized by Sigma-Aldrich (Merck KGaA, Darmstadt, Germany). qPCR was performed in triplicate using $1 \mu 1$ diluted complementary (c)DNA template (400 ng) in a $\mu 1$ total volume of $10 \mu \mathrm{l}$. Reactions were performed in a 384-well plate format using the ABI Viia7 Real-time PCR system (Thermo Fisher Scientific, Inc.) and Sensifast SYBR Lo-ROX Master Mix (Bioline; Meridian Life Science, Inc., Memphis, TN, USA). A third-step thermocycling protocol was used, with an initial hold at $95^{\circ} \mathrm{C}$ for $2 \mathrm{~min}$ to activate the polymerase, followed by $95^{\circ} \mathrm{C}$ 
Table II. Primers used for polymerase chain reaction analysis.

\begin{tabular}{|c|c|c|}
\hline Primer name & Primer sequence $\left(5^{\prime}-3^{\prime}\right)$ & Product length (bp) \\
\hline COL1A1-S & TCAGCTTTGTGGACCTCCG & 197 \\
\hline COL1A1-A & ATTGCATTGCACGTCATCG & \\
\hline ACTN1-S & AGGACACCTTCATCGTACATACCAT & 198 \\
\hline ACTN1-A & CCTGAGGCGTGATGGTTGTATAG & \\
\hline VCL-S & GTTGGAAAAGAGACTGTTCAGACCA & 171 \\
\hline VCL-A & CCTAGAGCCGTCAATGAGGTAATC & \\
\hline CAV1-S & GCAGACGAGGTGACTGAGAAGC & 224 \\
\hline CAV1-A & AAGATCGTAGACAACAAGCGGTAA & \\
\hline MYL9-S & АСтTTTCтTCTCTGCAGCAGGG & 253 \\
\hline MYL9-A & ATACTCGTCTGTGGGGTTCTTCC & \\
\hline THBS1-S & TTCCTGATGGTGAATGCTGCC & 158 \\
\hline THBS1-A & GCCCTCGCATCTGTTGTTGA & \\
\hline COL3A1-S & GTTTCTTCTCACССТTCTTCATCC & 247 \\
\hline COL3A1-A & AGGCTGTGGGCATATTGCA & \\
\hline SDC4-S & CCCTCAGAGCCCAAGGAACT & 179 \\
\hline SDC4-A & AAGAGGATGCCTACCACGCC & \\
\hline CD44-S & ATCCCTCCGTTTCATCCAGC & 226 \\
\hline CD44-A & CATGGTGGGTAAGGTACTGTTGAA & \\
\hline FDPS-S & AGGAGGTCCTAGAGTACAATGCC & 195 \\
\hline FDPS-A & TGAGGGAAGAGTCCATGATGTC & \\
\hline IDI1-S & CCATTAAGTAACCCAGGCGAG & 162 \\
\hline IDI1-A & ACCATCAGATTGGGCCTTGT & \\
\hline CYP51-S & ATTTGGAGCTGGGCGTCAT & 262 \\
\hline CYP51-A & TTCTCAGAGGCTTCATTCTTTGC & \\
\hline SQLE-S & ACCCGGAAGTGATCATCGTG & 203 \\
\hline SQLE-A & ATGGGCATTGAGACCTTCTACTGT & \\
\hline SPP1-S & TGTCCTCTGAAGAAAAGGATGACTT & 238 \\
\hline SPP1-A & TCGACTGTAGGGACGATTGGAG & \\
\hline GAPDH-S & GCCTTCCGTGTTCCTACC & 183 \\
\hline GAPDH-A & AGAGTGGGAGTTGCTGTTG & \\
\hline
\end{tabular}

S, sense; A, antisense; COL1A1, collagen type $1 \alpha 1$ chain; ACTN1, actin $\alpha 1$; VCL, vinculin; CAV1, caveolin 1; MYL9, myosin light chain 9; THBS1, thrombospondin 1; SDC4, syndecan 4; FDPS, farnesyl diphosphate synthase; IDI1, isopentyl-diphosphate $\delta$ isomerase 1; CYP51, cytochrome P 450 family 51; SQLE, squalene epoxidase; SPP, secreted phosphoprotein 1.

for $2 \mathrm{~min}, 40$ cycles of $95^{\circ} \mathrm{C}$ for $5 \mathrm{sec}, 60^{\circ} \mathrm{C}$ for $20 \mathrm{sec}, 72^{\circ} \mathrm{C}$ for $20 \mathrm{sec}$. The $2^{-\Delta \Delta \mathrm{Cq}}$ method was used for data normalization (19). GAPDH was used as the housekeeping gene.

Analysis of transcriptome sequencing results. Sequencing data were checked for sequencing quality using FASTQC (http://www.bioinformatics.babraham.ac.uk/projects/fastqc/). Adaptors and poor-quality sequences were then removed using Trim Galore v0.3.7 (http://www.bioinformatics.babraham. ac.uk/projects/trim_galore/). Pairs of trimmed reads were aligned against the human genome (hg19 build) using Tophat (v 2.1; https://ccb.jhu.edu/software/tophat/index.shtml).

Mapped fragments per kilobase of transcript per million (FPKM) of each gene or transcript variant were calculated using Cufflink software (http://cole-trapnell-lab.github. io/cufflinks/). Cuffdiff software produces the P-value and q-value. The number of DEGs screened by the Q-value may be too small for enrichment analysis. More DEGs may be obtained by using the P-value, which is conducive to subsequent enrichment analysis. Furthermore, certain randomly emerging DEGs can be filtered out by enrichment analysis to avoid their influence on the experimental result. Therefore, the P-value was selected as a screening index, with a cutoff criterion of $\mathrm{P}<0.05$, which was used to screen for DEGs. Functional enrichment analysis of DEGs was performed using Kyoto Encyclopedia of Genes and Genomes (KEGG) pathway analysis (www.genome.jp/kegg) and gene ontology (GO) analysis (http://www.geneontology.org/).

Statistical analysis. All data were analyzed using SPSS 18.0 software (SPSS, Inc., Chicago, IL, USA). Fisher's exact test was used to identify the DEGs. The Benjamini-Hochberg 

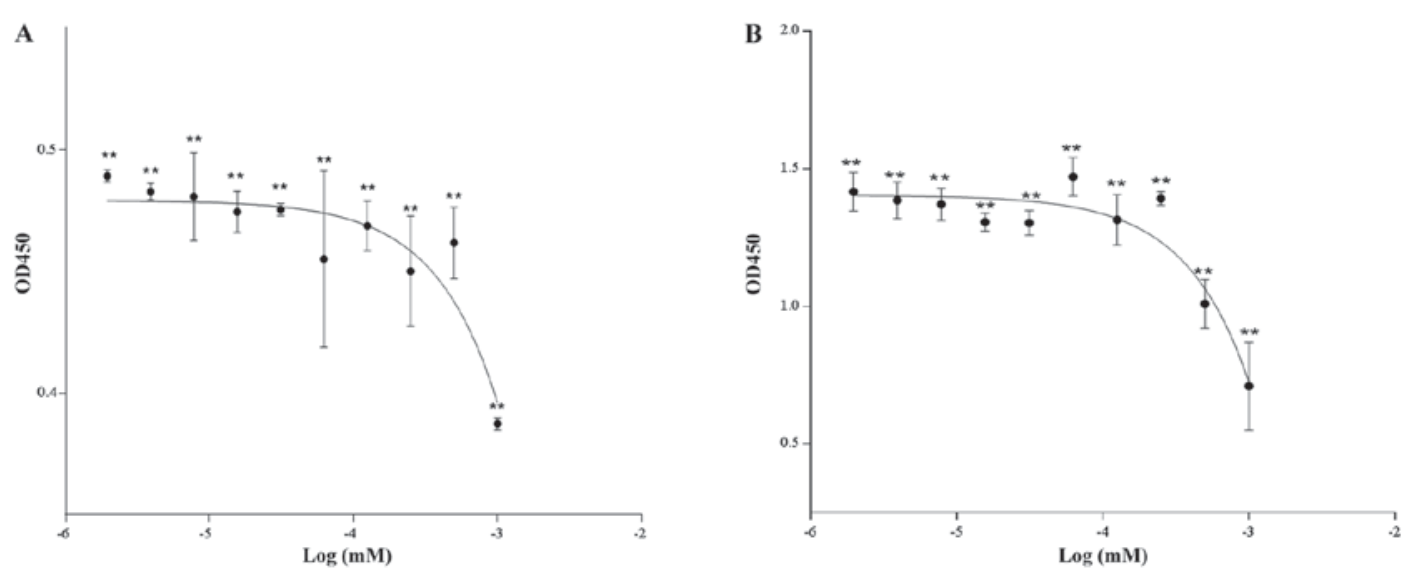

Figure 1. Effect of MEHP on cell proliferation. Cell proliferation curve following exposure to MEHP for (A) $24 \mathrm{~h}$ and (B) $48 \mathrm{~h}$. ** $\mathrm{P}<0.01 \mathrm{vs}$. control (0 mM). MEHP, mono-(2-ethylhexyl) phthalate; OD, optical density.

correction was used to control for false-positives. Normally distributed data are presented as the mean \pm standard deviation, while a Kruskal-Wallis test was used to analyze data with an abnormal distribution, which are presented as the median and interquartile range (Q). Shapiro-Wilk test was used to examine whether data were normally distributed. First, one-way analysis of variance was used to determine significant differences among all groups, including one control group and two treated groups. If the difference was statistically significant, a Fisher's least-significant differences test was used for further mutual and random comparison among the three groups. $\mathrm{P}<0.05$ was considered to indicate a statistically significant difference.

\section{Results}

Analysis of apoptosis. TM4 cells treated with different concentrations of MEHP for $48 \mathrm{~h}$ were analyzed using flow cytometry to detect apoptosis. The results indicated that MEHP at concentrations of $\leq 1 \times 10^{-4} \mathrm{~mol} / 1$ did not significantly affect the viability of the cells $\left(\chi^{2}=7.421 ; \mathrm{P}=0.06\right.$; Table I). Therefore, MEHP was used at this non-toxic concentration range for the subsequent cell proliferation assays.

Cell proliferation. The cell growth curves indicated that the differences in cell proliferation among the groups were more marked after $48 \mathrm{~h}$ of exposure compared with those at $24 \mathrm{~h}$. The cell proliferation rates of the con 1 and con 3 groups were significantly lower than those in the other groups (Fig. 1). In order to better reflect the differences in phenotypic changes of the cells, con 1 and con 3 were used as the high and low doses, respectively, in the transcriptome sequencing experiments and the exposure time was set at $48 \mathrm{~h}$.

Transcriptome sequencing and DEG analysis. To determine the effects of MEHP on Sertoli cells, transcriptome sequencing was performed using a cDNA library generated from an equal amount of RNA isolated from the control or MEHP-treated TM4 cells. An average of 10,947 genes (with a required FPKM value of $>0.7$ ) were detected among the different groups.

A total of 528 and 269 genes were identified to be upregulated in the con 1 and con3 group cells, respectively, compared with the control group (DMSO); in addition, 148 and
173 genes were downregulated, respectively (Fig. 2). Among these genes, 165 were consistently upregulated and 45 were consistently downregulated in the two MEHP-treated groups (con1 and con3). These 210 DEGs were regarded as candidates for further investigation.

Enrichment analysis of DEGs. Functional enrichment analysis of DEGs was performed using the GO and KEGG methods. GO analysis of the con1 group provided significant enrichment of DEGs in 119 terms in the biological process (BP), 20 in the cellular component (CC) and 16 in the molecular function $(\mathrm{MF})$ category $(\mathrm{P}<0.01)$. For the con3 group, the DEGs were significantly enriched in $116 \mathrm{BP}, 6 \mathrm{CC}$ and $12 \mathrm{MF}$ terms $(\mathrm{P}<0.01)$. The top $30 \mathrm{GO}$ categories with significant enrichment of DEGs (con1 and con3 vs. DMSO group) are presented in Fig. 3A and B, respectively. Based on the level of significance, the top term was 'extracellular region' in the CC domain in the con1 and con3 groups.

In the KEGG analysis, 22 signaling pathways were significantly enriched by the DEGs from the con 1 group $(\mathrm{P}<0.05$; Fig. 4A). For the con 3 group, 10 signaling pathways were significantly enriched by the DEGs $(\mathrm{P}<0.05$; Fig. 4B). The pathways were mainly associated with tumors, steroid synthesis and cell adhesion. According to the statistical significance, the top three pathways were 'focal adhesion', 'extracellular matrix (ECM)-receptor interaction' and 'terpenoid backbone biosynthesis.

Compared with the control group, there were eight common enriched pathways in the high- and low-dose groups, including 'terpenoid backbone biosynthesis', 'focal adhesion', 'ECM-receptor interaction', 'pathways in cancer', 'colorectal cancer', 'transforming growth factor- $\beta$ signaling pathway', 'vascular smooth muscle contraction' and 'axon guidance'.

$R T$ - $q P C R$ validation of transcriptome sequencing. To validate the results of the transcriptome sequencing, transcripts of 14 key DEGs associated with the enriched KEGG pathways were detected using RT-qPCR (Fig. 5). The analysis confirmed the aberrant expression of collagen type I $\alpha 1$ chain (COL1A1), actinin $\alpha 1$ (ACTN1), vinculin (VCL), caveolin 1 (CAV1) and myosin light chain 9 (MYL9) for the 'focal adhesion' pathway, thrombospondin 1 (THBS1), COL3A1, secreted 


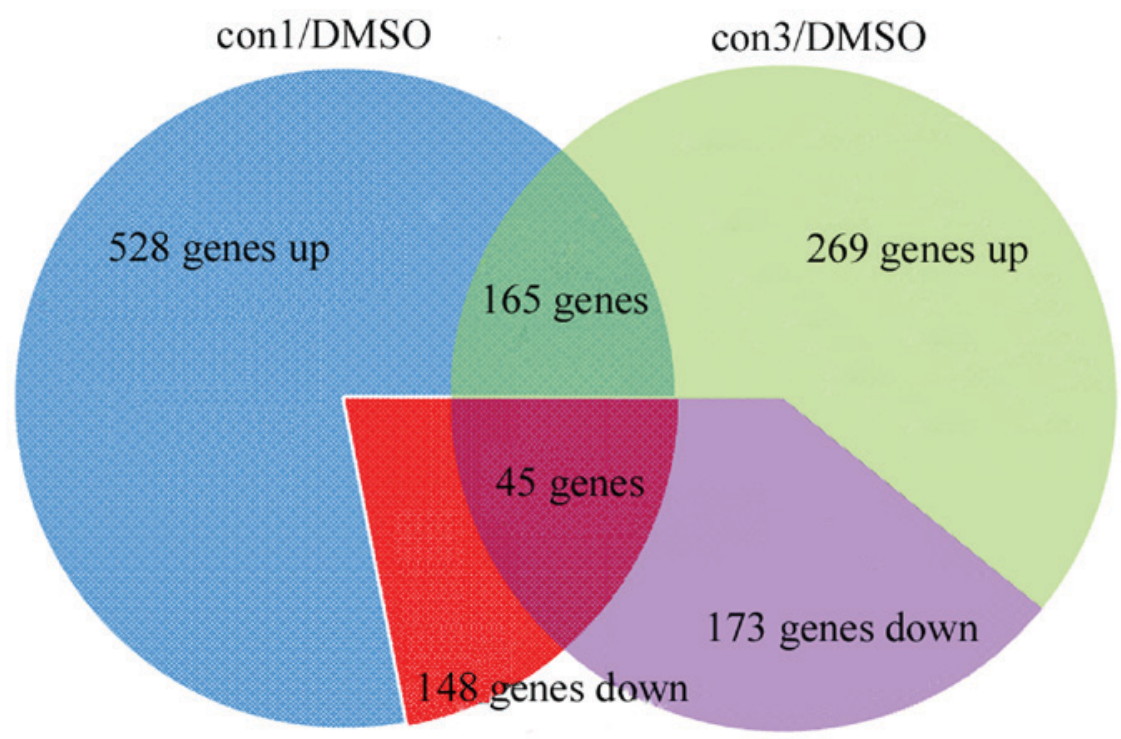

Figure 2. Composition of DEGs in two samples with different concentrations used for transcriptome sequencing. DEGs, differentially expressed genes; DMSO, dimethyl sulfoxide; up, upregulated; down, downregulated; con1, MEHP at $1 \times 10^{-3} \mathrm{mmol} / 1$; con2, MEHP at $5 \times 10^{-4} \mathrm{mmol} / 1$.

A
용

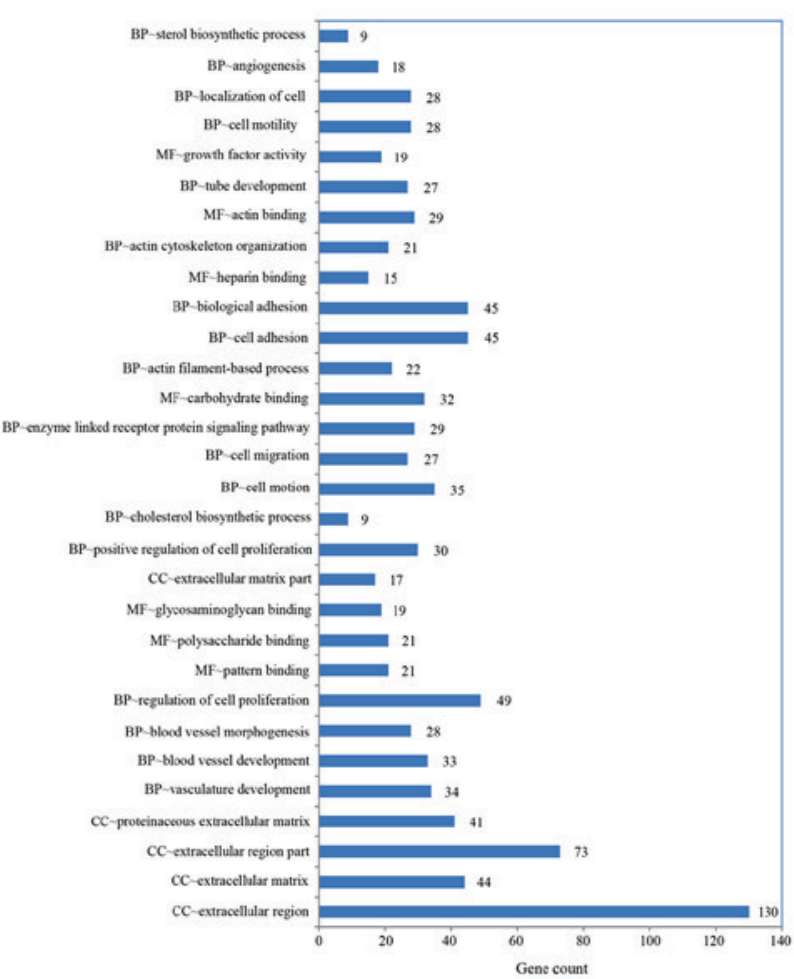

B

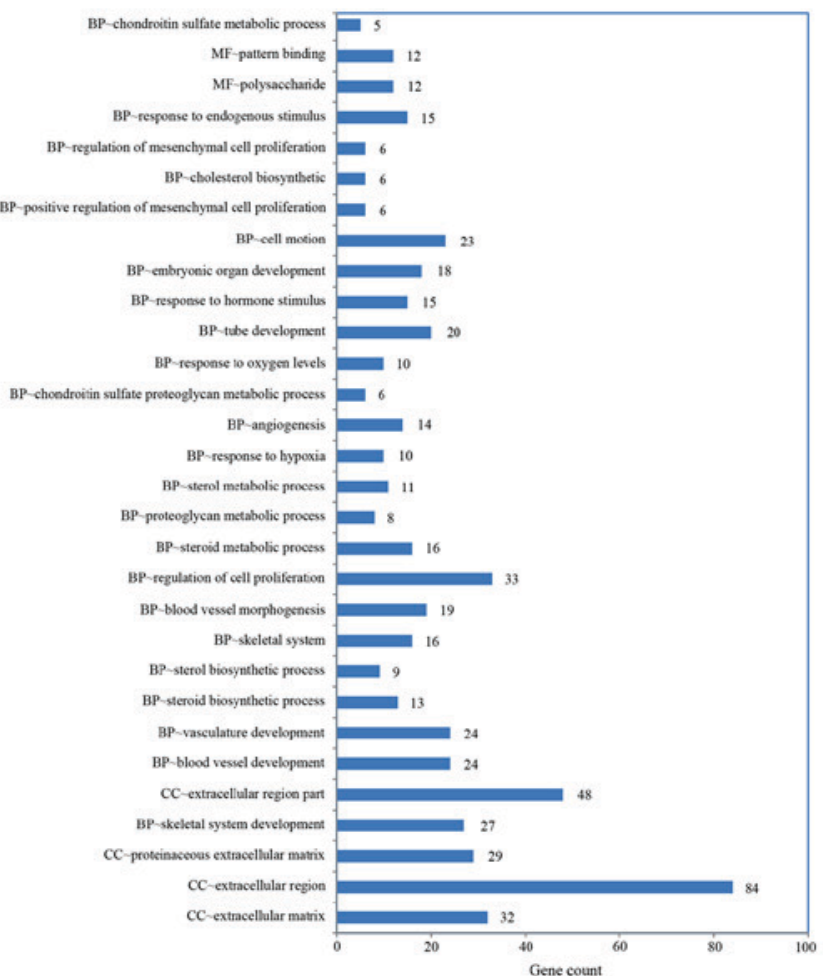

Figure 3. GO analysis of DEGs. (A) GO categories of the con1 vs. DMSO group DEGs. (B) GO categories of the con3 vs. DMSO group DEGs. GO, gene ontology; DEGs, differentially expressed genes; DMSO, dimethyl sulfoxide; MF, molecular function; BP, biological process; CC, cellular component; con 1, MEHP at $1 \times 10^{-3} \mathrm{mmol} / 1 ;$ con3, MEHP at $2.5 \times 10^{-4} \mathrm{mmol} / 1$.

phosphoprotein 1 (SPP1), syndecan 4 (SDC4) and CD44 for the 'ECM-receptor interaction', and farnesyl diphosphate synthase (FDPS), isopentenyl-diphosphate $\delta$ isomerase 1 (IDI1), cytochrome P450 family 51 (CYP51) and squalene epoxidase (SQLE) for 'terpenoid backbone biosynthesis and steroid biosynthesis'. Please clarify whether this only applies to SQLE.

The mRNA levels of COL1A, VCL, ACTN1, CAV1, MYL9, THBS1, COL3A, SDC4, CD44 and SPP1 were significantly decreased in the low-does (con3) and high-dose (con1) groups. However, four genes, FDPS, IDI1, CYP51 and SQLE, were increased in the low-dose group and decreased in the high-dose group.

\section{Discussion}

In the last 70 years, the use of phthalates has changed the materials science substantially, which has made exposure to 
A

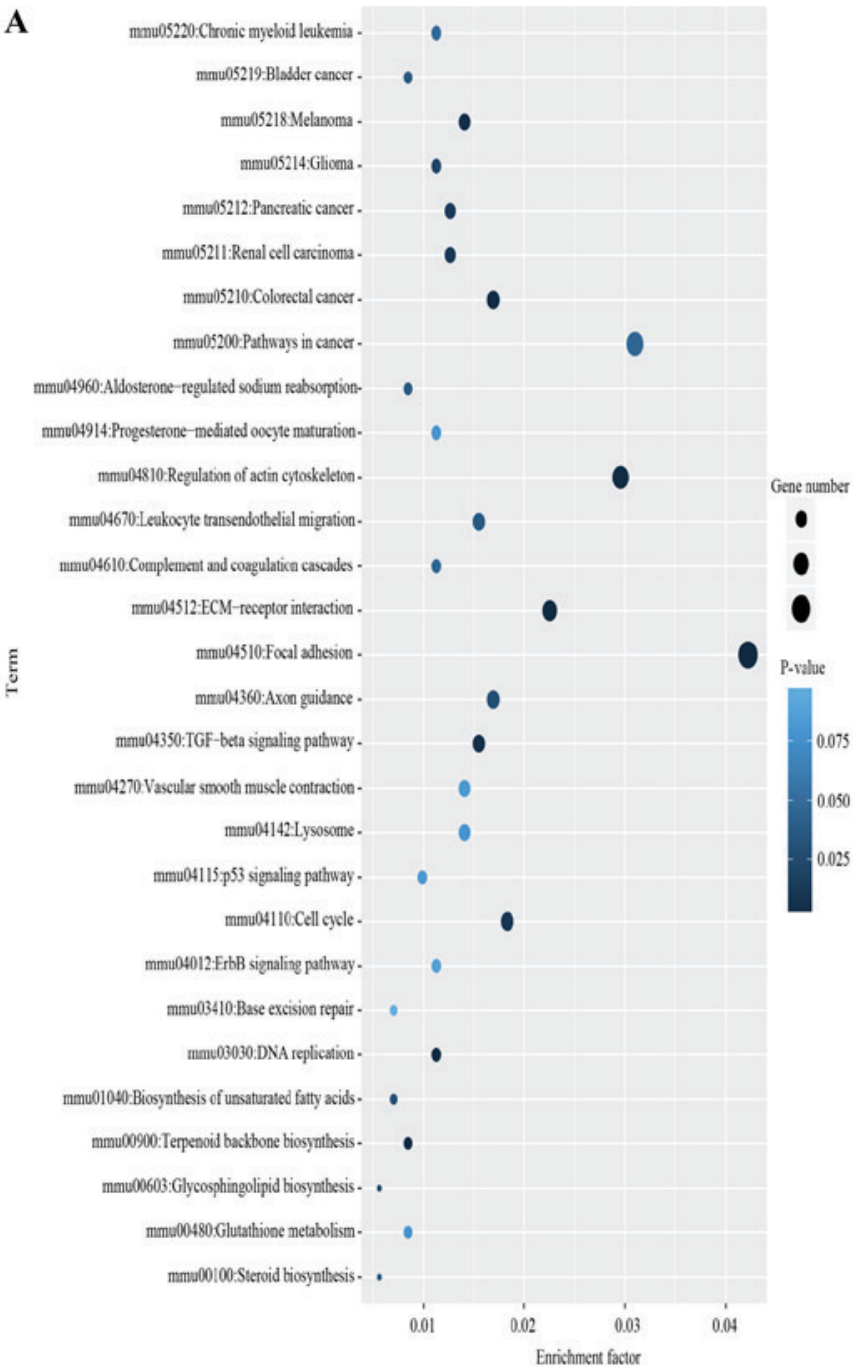

B

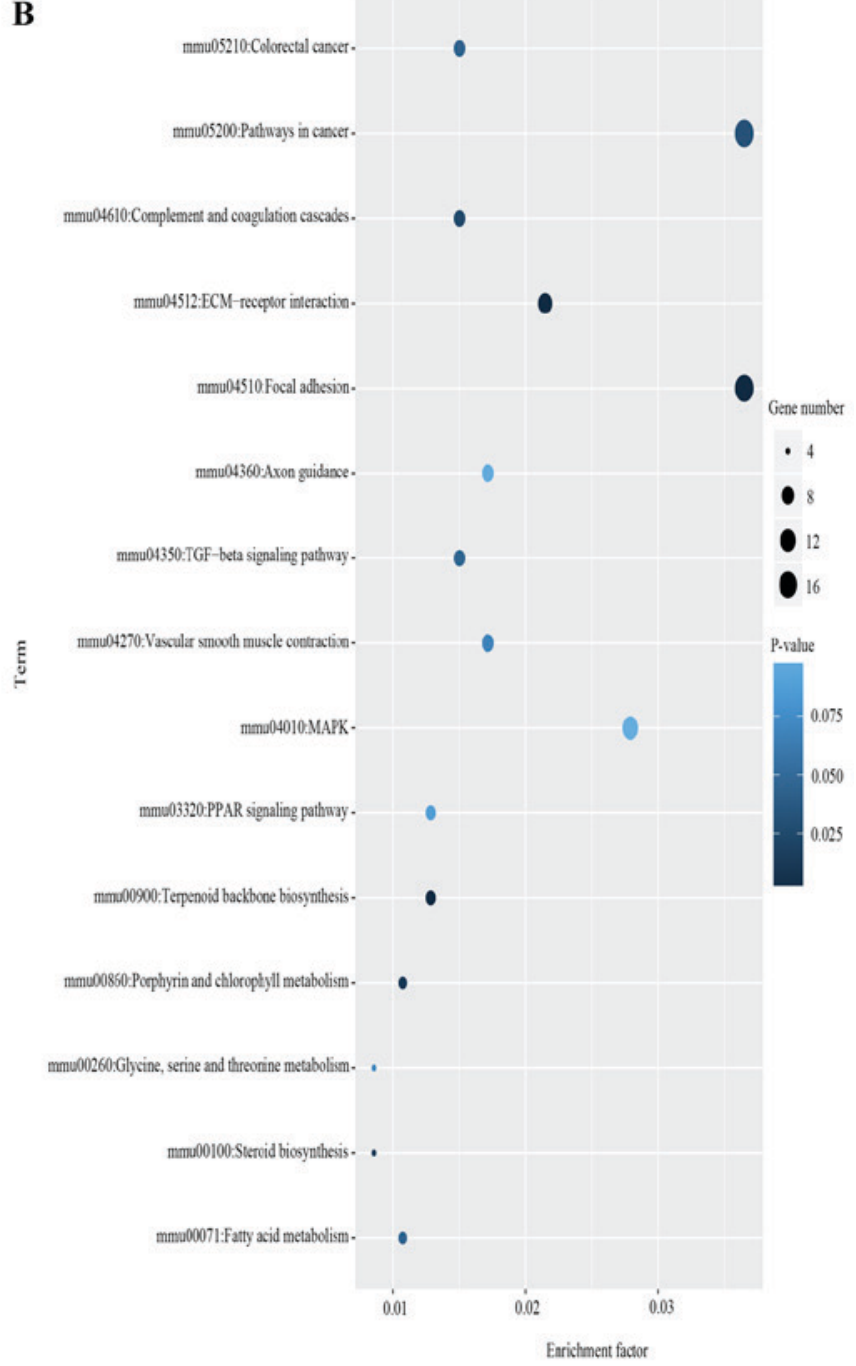

Figure 4. Scatterplot for most enriched KEGG pathways of DEGs in the MEHP-treated group compared with control cells. (A) Differentially enriched KEGG pathways in the con1 group compared with the control group. (B) Differentially enriched KEGG pathways in the con3 group compared with the control group. Enrichment factor was the ratio of the number of DEGs to the total gene number. Smaller P-values indicate a higher degree of enrichment. KEGG, Kyoto Encyclopedia of Genes and Genomes; DEGs, differentially expressed genes; MEHP, mono-(2-ethylhexyl) phthalate; ECM, extracellular matrix; TGF, transforming growth factor; MAPK, mitogen-activated protein kinase; mmu, Mus musculus; con 1, con1, MEHP at $1 \times 10^{-3} \mathrm{mmol} / 1$; con3, MEHP at 2.5x10 ${ }^{-4} \mathrm{mmol} / 1$.

phthalates unavoidable. In view of the harm that phthalates may cause to the body, particularly in sensitive populations, including infants and young children, the damaging effects of phthalates have received increasing attention (20-24). However, the internal exposure of the human body to phthalates was not as high as expected. Studies have reported that the mean concentrations of MEHP in the cord blood of neonates, the group considered to be most sensitive to the developmental and reproductive toxicity of phthalates, were $2 \times 10^{-6}$ and $1.1 \times 10^{-8} \mathrm{M}(4,25)$. Although exposure to small amounts does not directly cause cell death, it may have sub-lethal cytological effects, including genetic damage and malfunction of cellular metabolism (26). MEHP is the major toxic metabolite of DEHP and is known to have reproductive and developmental toxicity (27-29); thus, in the present study, a low exposure dose of MEHP that was not lethal to TM4 cells was used in order to realistically mimic human internal exposure to DEHP, which is the most widely used phthalate. The appropriate dose of MEHP, which was not lethal to TM4 cells, was determined in a cell apoptosis assay. The concentration and exposure time used in the transcriptome sequencing experiment were determined from the results of a cell proliferation assay. Transcriptome sequencing of TM4 cells was performed following treatment with MEHP or control treatment. The combination of next-generation transcriptome sequencing technology and bioinformatics analysis provides a useful method to analyze the mechanisms of action of toxic chemical agents, and to identify potential hazardous compounds. To the best of our knowledge, the present study was the first to use transcription sequencing to investigate gene expression changes in the TM4 Sertoli cell line following treatment with MEHP.

Transcriptomics approaches have previously been used to investigate the toxic effects of DEHP (30). Studies have revealed that phthalates have effects on peroxisome proliferator-activated receptors (PPARs), tumor necrosis factor signaling pathways, calcium binding and numerous myosin proteins. In the present study, 165 genes were upregulated and 45 were downregulated in the two MEHP-treated groups of TM4 cells. The genes with the greatest change in expression 


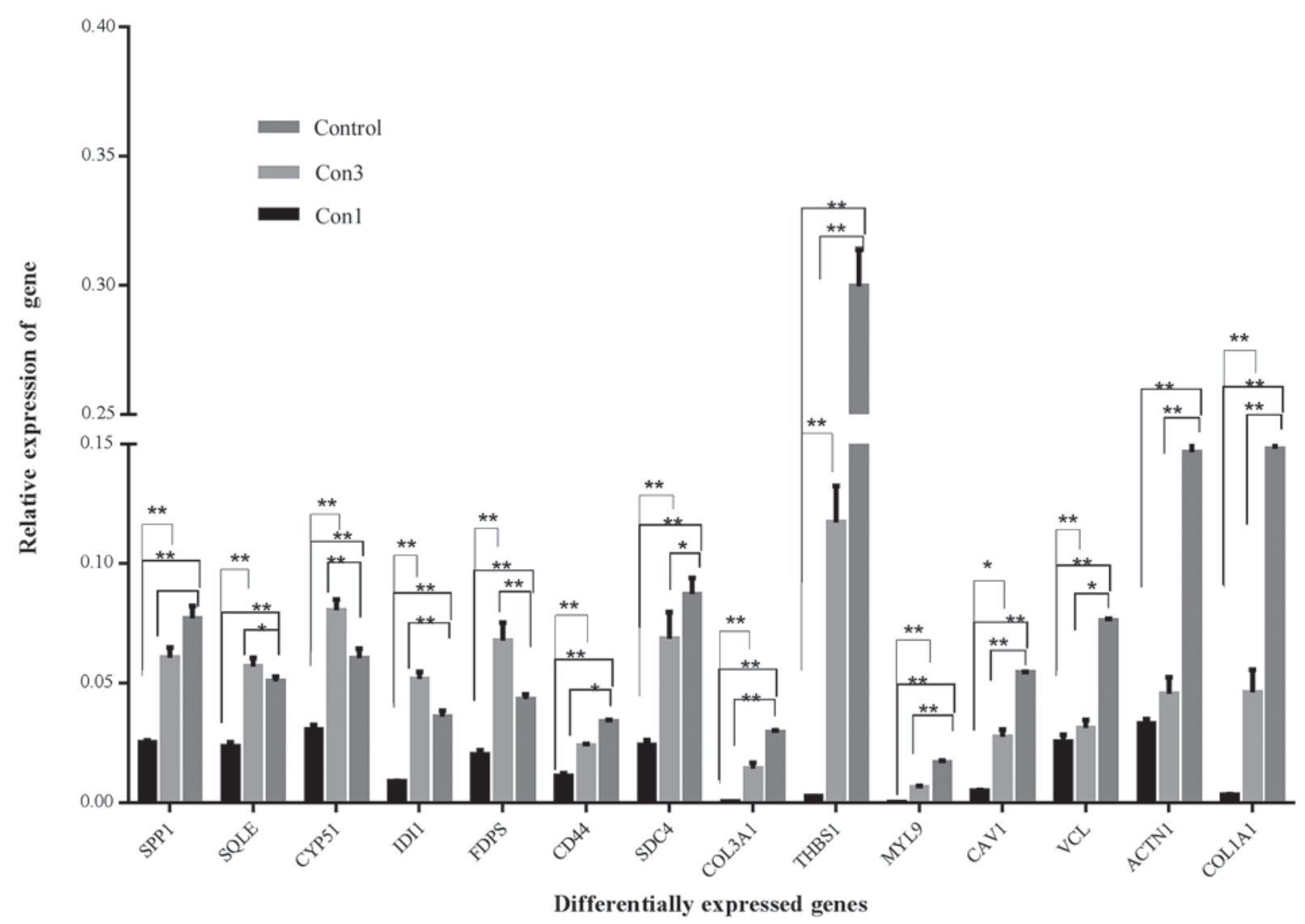

Figure 5. mRNA expression of 14 genes in the MEMP-treated TM4 cells. ${ }^{*} \mathrm{P}<0.05,{ }^{* *} \mathrm{P}<0.01$. MEHP, mono-(2-ethylhexyl) phthalate; con1, MEHP at $1 \times 10^{-3} \mathrm{mmol} / 1$; con3, MEHP at 2.5x10 $0^{-4} \mathrm{mmol} / 1$; COL1A1, collagen type $1 \alpha 1$ chain; ACTN1, actin $\alpha 1$; VCL, vinculin; CAV1, caveolin 1; MYL9, myosin light chain 9; THBS1, thrombospondin 1; SDC4, syndecan 4; FDPS, farnesyl diphosphate synthase; IDI, isopentyl-diphosphate $\delta$ isomerase 1; CYP51, cytochrome P 450 family 51; SQLE, squalene epoxidase; SPP, secreted phosphoprotein 1.

out of the 210 DEGs may potentially be used as biomarkers of DEHP-induced damage and as a useful tool to further improve the identification of developmental toxicants (31). The DEGs of the high- and low-dose groups had 8 pathways in common. In the GO analysis, the terms with the highest significance, in the con 1 and con 3 groups, was 'extracellular region' in the CC domain. In the KEGG analysis, the top three pathways $(\mathrm{P}<0.01)$ were 'focal adhesion', 'regulation of actin cytoskeleton' and 'ECM receptor interaction'. The results were consistent for the responses of TM4 cells to $1 \times 10^{-3}$ and $2.5 \times 10^{-4} \mathrm{mmol} / 1 \mathrm{MEHP}$. In the study by Nardelli et al (32), 13 DEGs associated with PPAR and cholesterol biosynthesis signaling pathways were identified using a gene expression microarray. The present study provides evidence supporting the roles of genes associated with 'focal adhesion', 'regulation of actin cytoskeleton' and 'ECM-receptor interaction' as the key functional genes that mediate the effects of low-dose MEHP in Sertoli cells. Furthermore, the RT-qPCR results confirmed that the expression of genes with roles in 'focal adhesion' and 'ECM-receptor interaction' was decreased with the levels of MEHP exposure increasing, indicating a dose-response effect.

KEGG analysis revealed that the 'focal adhesion' pathway was enriched in the largest number of DEGs in the high- and low-dose groups, and RT-qPCR analysis confirmed that the gene expression of COL1A1, ACTN1, VCL, CAV1 and MYL9 was decreased following MEHP treatment in a dose-dependent manner. Ectoplasmic specialization (ES) is a unique cellular structure that maintains cell shape and connections between Sertoli cells. Actinin, fimbrin, espin and vinculin are all involved in ES $(33,34)$. It was previously reported that MEHP is able to destroy the ES between rat Sertoli cells and spermatogenic cells, causing a release of the immature germ cells into the seminiferous tubule, which weakens reproductive function $(35,36)$. The present study indicated that the expression of the genes that encode actinin and vinculin was decreased by MEHP, which was similar to the results of a previous study, in which Syrian hamster embryo cells were exposed to $50 \mu \mathrm{M}$ DEHP for $24 \mathrm{~h}$, revealing that the downregulated genes were associated with focal adhesion or cell junctions (37). This indicates that MEHP may affect the ECM-receptor interaction and focal adhesions to disrupt intercellular junctions and the formation of fissures between Sertoli cells and spermatogenic cells, resulting in the loss of spermatogenic cells from the seminiferous epithelium.

Previous studies have reported that target genes altered by phthalates are involved in several important signaling pathways, including steroid synthesis, as well as lipid and cholesterol homeostasis $(38,39)$. Testosterone is the major androgen and anabolic hormone, with an important role in the growth and development of male animals. It has been previously demonstrated that DEHP is able to mimic or antagonize the actions of steroid hormones (40), and the effects of DEHP on testosterone and estrogen-like activity 
have also been reported (41). Considering the fundamental role of steroid hormones in reproductive and developmental functions, an imbalance in the synthesis and/or signaling of these hormones may adversely affect different aspects of sexual development (42). Of note, in the present study, the enrichment of two pathways, 'terpenoid backbone biosynthesis' and 'steroid biosynthesis', was higher in the low-dose MEHP group than the high-dose group and the control group, indicating that different doses of MEHP may exhibit opposing effects on the synthesis of steroidal compounds in Sertoli cells, which is potentially associated with the excitatory effect of MEHP. Biphasic effects of di (n-butyl) phthalate were observed on cholesterol side-chain cleavage enzyme and $3 \beta$-hydroxysteroid dehydrogenase mRNA, which were generally increased at a low dose of $10 \mathrm{mg} / \mathrm{kg}$, and at higher doses (50-400 mg/kg), an apparent dose-dependent decrease was obtained (43). Hormesis is a concept describing a dose-response association where there is a stimulation at a low dose and inhibition at a high dose (44). Hormetic effects are considered as an adaptive response to a moderate stress induced by the stimulus (45). A study has proved that the developmental toxicity of DEHP may be hermetic by using a score test (46). In this light, the present results suggest a hormetic-like biphasic dose-response association of MEHP in Sertoli cells. Cell signaling-mediated bidirectional control of gene expression has been considered as the major hormetic mechanism triggered by exposure to xenobiotics (47). This indicates that 'terpenoid backbone biosynthesis' and 'steroid biosynthesis' signaling pathways were activated in response to this low level of exposure. This indicates that MEHP may affect testosterone synthesis via multiple mechanisms to cause reproductive toxicity.

The results of the present study demonstrated that the mechanisms of the toxicity of MEHP are complex, including effects on reproduction, development and metabolism involving a numerous different genes, cellular processes and signaling pathways. The interaction of these factors ultimately leads to the complex toxic effects of MEHP. Although the present study is limited to gene expression changes, the results of the present study provide a foundation for further examination of the toxic effects of low-level phthalate exposure. More gene and protein expression validation and functional analyses should be performed to fully elucidate the molecular mechanisms of phthalate toxicity.

\section{Acknowledgements}

Not applicable.

\section{Funding}

This work was supported by funding from the National Natural Science Foundation of China (grant no. 81202208).

\section{Availability of data and materials}

The datasets generated and/or analyzed during the current study are not publicly available due to ongoing research; however, data are available from the corresponding author on reasonable request.

\section{Authors' contributions}

LW was responsible for experimental design, implementation and writing of article. TD was responsible for experimental data processing. SL was responsible for experimental design and revision of the article. YL was responsible for experimental design and revision of the article. All authors have read and approved the manuscript.

\section{Ethics approval and informed consent}

Not applicable.

\section{Patient consent for publication}

Not applicable.

\section{Competing interests}

The authors declare that they have no competing interests.

\section{References}

1. Schettler T: Human exposure to phthalates via consumer products. Int J Androl 29: 134-139; discussion 181-185, 2006.

2. Markarian J: PVC additives-What lies ahead? Plast Addit Compound 9: 22-25, 2007.

3. Högberg J, Hanberg A, Berglund M, Skerfving S, Remberger M, Calafat AM, Filipsson AF, Jansson B, Johansson N, Appelgren M and Håkansson $\mathrm{H}$ : Phthalate diesters and their metabolites in human breast milk, blood or serum, and urine as biomarkers of exposure in vulnerable populations. Environ Health Perspect 116: 334-339, 2008.

4. Latini G, De Felice C, Presta G, Del Vecchio A, Paris I, Ruggieri F and Mazzeo P: In utero exposure to di-(2-ethylhexyl)phthalate and duration of human pregnancy. Environ Health Perspect 111: 1783-1785, 2003.

5. Latini G, De Felice C, Presta G, Del Vecchio A, Paris I, Ruggieri F and Mazzeo P: Exposure to Di(2-ethylhexyl)phthalate in humans during pregnancy. A preliminary report. Biol Neonate 83: 22-24, 2003.

6. Swan SH: Prenatal phthalate exposure and anogenital distance in male infants. Environ Health Perspect 114: A88-A89, 2006.

7. Fay M, Donohue JM and De Rosa C: ATSDR evaluation of health effects of chemicals. VI. Di(2-ethylhexyl)phthalate. Agency for toxic substances and disease registry. Toxicol Ind Health 15: 651-746, 1999.

8. Lyche JL, Gutleb AC, Bergman A, Eriksen GS, Murk AJ, Ropstad E, Saunders M and Skaare JU: Reproductive and developmental toxicity of phthalates. J Toxicol Environ Health B Crit Rev 12: 225-249, 2009.

9. Fratantoni JC: Review: The platelet storage lesion: Possible role of plasticizers? Blood Cells 18: 435-440; discussion 441-443, 1992.

10. Hayes DN and Kim WY: The next steps in next-gen sequencing of cancer genomes. J Clin Invest 125: 462-468, 2015.

11. Wang Q, Lu Q and Zhao H: A review of study designs and statistical methods for genomic epidemiology studies using next generation sequencing. Front Genet 6: 149, 2015.

12. Fiorini C, Tilloy-Ellul A, Chevalier S, Charuel C and Pointis G: Sertoli cell junctional proteins as early targets for different classes of reproductive toxicants. Reprod Toxicol 18: 413-421, 2004.

13. Rodriguez-Sosa JR, Bondareva A, Tang L, Avelar GF, Coyle KM, Modelski M, Alpaugh W, Conley A, Wynne-Edwards K, França LR, et al: Phthalate esters affect maturation and function of primate testis tissue ectopically grafted in mice. Mol Cell Endocrinol 398: 89-100, 2014.

14. Sjöberg P, Bondesson U, Kjellen L, Lindquist NG, Montin G and Plöen L: Kinetics of di-(2-ethylhexyl) phthalate in immature and mature rats and effect on testis. Acta Pharmacol Toxicol (Copenh) 56: 30-37, 1985. 
15. Sjöberg P, Lindqvist NG and Plöen L: Age-dependent response of the rat testes to di(2-ethylhexyl) phthalate. Environ Health Perspect 65: 237-242, 1986.

16. Chapin RE, Gray TJ, Phelps JL and Dutton SL: The effects of mono-(2-ethylhexyl)-phthalate on rat Sertoli cell-enriched primary cultures. Toxicol Appl Pharmacol 92: 467-479, 1988.

17. Galdieri M, Ziparo E, Palombi F, Russo MA and Stefanini M: Pure sertoli cell cultures: A new model for the study of somatic-Germ cell interactions. J Androl 2: 249-254, 1981

18. van Dijk EL, Auger H, Jaszczyszyn Y and Thermes C: Ten years of next-generation sequencing technology. Trends Genet 30: 418-426, 2014

19. Livak KJ and Schmittgen TD: Analysis of relative gene expression data using real-time quantitative PCR and the 2(-Delta Delta C(T)) method. Methods 25: 402-408, 2001

20. Casas L, Fernández MF, Llop S, Guxens M, Ballester F, Olea N, Irurzun MB, Rodríguez LS, Riaño I, Tardón A, et al: Urinary concentrations of phthalates and phenols in a population of Spanish pregnant women and children. Environ Int 37: 858-866, 2011.

21. Frederiksen H, Aksglaede L, Sorensen K, Skakkebaek NE, Juul A and Andersson AM: Urinary excretion of phthalate metabolites in 129 healthy Danish children and adolescents: Estimation of daily phthalate intake. Environ Res 111: 656-663, 2011

22. Guo Y and Kannan K: Comparative assessment of human exposure to phthalate esters from house dust in China and the United States. Environ Sci Technol 45: 3788-3794, 2011.

23. Romero-Franco M, Hernández-Ramírez RU, Calafat AM, Cebrián ME, Needham LL, Teitelbaum S, Wolff MS and López-Carrillo L: Personal care product use and urinary levels of phthalate metabolites in Mexican women. Environ Int 37: 867-871, 2011

24. Woodruff TJ, Zota AR and Schwartz JM: Environmental chemicals in pregnant women in the United States: NHANES 2003-2004. Environ Health Perspect 119: 878-885, 2011.

25. Lin S, Ku HY, Su PH, Chen JW, Huang PC, Angerer J and Wang SL: Phthalate exposure in pregnant women and their children in central Taiwan. Chemosphere 82: 947-955, 2011.

26. Midic U, Vincent KA, VandeVoort CA and Latham KE: Effects of long-term endocrine disrupting compound exposure on Macaca mulatta embryonic stem cells. Reprod Toxicol 65: 382-393, 2016.

27. Moss EJ, Cook MW, Thomas LV and Gray TJ: The effect of mono-(2-ethylhexyl) phthalate and other phthalate esters on lactate production by Sertoli cells in vitro. Toxicol Lett 40 : 77-84, 1988.

28. Lamb JC IV and Chapin RE: Testicular and germ cell toxicity: In vitro approaches. Reprod Toxicol 7 (Suppl 1): S17-S22, 1993.

29. Davis BJ, Weaver R, Gaines LJ and Heindel JJ: Mono-(2-ethylhexyl) phthalate suppresses estradiol production independent of FSH-cAMP stimulation in rat granulosa cells. Toxicol Appl Pharmacol 128: 224-228, 1994.

30. Stenz L, Escoffier J, Rahban R, Nef S and Paoloni-Giacobino A Testicular dysgenesis syndrome and long-lasting epigenetic silencing of mouse sperm genes involved in the reproductive system after prenatal exposure to DEHP. PLoS One 12: e0170441, 2017.

31. van Dartel DA, Pennings JL, Robinson JF, Kleinjans JC and Piersma AH: Discriminating classes of developmental toxicants using gene expression profiling in the embryonic stem cell test. Toxicol Lett 201: 143-151,2011.
32. Nardelli TC, Erythropel HC and Robaire B: Toxicogenomic screening of replacements for di(2-Ethylhexyl) phthalate (DEHP) using the immortalized TM4 sertoli cell line. PLoS One 10 e0138421, 2015.

33. Vogl AW, Pfeiffer DC and Redenbach DM: Ectoplasmic ('junctional') specializations in mammalian Sertoli cells: Influence on spermatogenic cells. Ann NY Acad Sci 637: 175-202, 1991.

34. Vogl AW, Pfeiffer DC, Mulholland D, Kimel G and Guttman J: Unique and multifunctional adhesion junctions in the testis: Ectoplasmic specializations. Arch Histol Cytol 63: 1-15, 2000.

35. Li LH, Jester WF Jr and Orth JM: Effects of relatively low levels of mono-(2-ethylhexyl) phthalate on cocultured Sertoli cells and gonocytes from neonatal rats. Toxicol Appl Pharmacol 153: 258-265, 1998

36. Yao PL, Lin YC and Richburg JH: Mono-(2-ethylhexyl) phthalate-induced disruption of junctional complexes in the seminiferous epithelium of the rodent testis is mediated by MMP2. Biol Reprod 82: 516-527, 2010.

37. Landkocz Y, Poupin P, Atienzar F and Vasseur P: Transcriptomic effects of di-(2-ethylhexyl)-phthalate in Syrian hamster embryo cells: An important role of early cytoskeleton disturbances in carcinogenesis? BMC Genomics 12: 524, 2011

38. Thompson CJ, Ross SM and Gaido KW: Di(n-butyl) phthalate impairs cholesterol transport and steroidogenesis in the fetal rat testis through a rapid and reversible mechanism. Endocrinology 145: 1227-1237, 2004.

39. Liu K, Lehmann KP, Sar M, Young SS and Gaido KW: Gene expression profiling following in utero exposure to phthalate esters reveals new gene targets in the etiology of testicular dysgenesis. Biol Reprod 73: 180-192, 2005.

40. Sharpe RM: Hormones and testis development and the possible adverse effects of environmental chemicals. Toxicol Lett 120: 221-232, 2001.

41. Akingbemi BT, Ge R, Klinefelter GR, Zirkin BR and Hardy MP Phthalate-induced Leydig cell hyperplasia is associated with multiple endocrine disturbances. Proc Natl Acad Sci USA 101: 775-780, 2004

42. Gunnarsson D, Leffler P, Ekwurtzel E, Martinsson G, Liu K and Selstam G: Mono-(2-ethylhexyl) phthalate stimulates basal steroidogenesis by a cAMP-independent mechanism in mouse gonadal cells of both sexes. Reproduction 135: 693-703, 2008.

43. Bello UM, Madekurozwa MC, Groenewald HB, Aire TA and Arukwe A: The effects on steroidogenesis and histopathology of adult male Japanese quails (Coturnix coturnix japonica) testis following pre-pubertal exposure to di(n-butyl) phthalate (DBP). Comp Biochem Physiol C Toxicol Pharmacol 166: 24-33, 2014.

44. Calabrese EJ and Baldwin LA: Hormesis: U-shaped dose responses and their centrality in toxicology. Trends Pharmacol Sci 22: 285-291, 2001

45. Calabrese EJ: Overcompensation stimulation: A mechanism for hormetic effects. Crit Rev Toxicol 31: 425-470, 2001.

46. Hunt D and Rai SN: Testing threshold and hormesis in a random effects dose-response model applied to developmental toxicity data. Biom J 47: 319-328, 2005.

47. Chen F, Liu SS, Yu M, Qu R and Wang MC: Blocking the entrance of AMP pocket results in hormetic stimulation of imidazolium-based ionic liquids to firefly luciferase. Chemosphere 132: 108-113, 2015. 